\title{
Statins: Hepatic Disease and Hepatotoxicity Risk
}

\author{
Raquel Cueto ${ }^{1}$, Pedro Valdivielso ${ }^{2}, \mathrm{M}^{\mathrm{a}}$ Isabel Lucena ${ }^{*}, 1, \mathrm{a}$, Carlota García-Arias ${ }^{2}$, Raúl J. Andrade ${ }^{3, a}$ \\ and Pedro González-Santos ${ }^{2}$
}

\author{
${ }^{1}$ Servicio de Farmacología Clínica, Hospital Clínico Universitario Virgen de la Victoria, Universidad de Málaga, Spain \\ ${ }^{2}$ Unidad de Lípidos, Medicina Interna, Hospital Clínico Universitario Virgen de la Victoria, Universidad de Málaga, \\ Spain \\ ${ }^{3}$ Unidad de Hepatología, Servicio de Aparato Digestivo, Hospital Universitario Virgen de la Victoria, School of \\ Medicine, Málaga, Spain \\ ${ }^{a}$ Centro de Investigación Biomédica en Red: Enfermedades Hepáticas y Digestivas (CIBERehd) - Málaga, Spain
}

\begin{abstract}
The hydroxy-methyl glutaryl coenzyme A (HMG-CoA) reductase inhibitors generally have a good safety profile and are one of the most prescribed drugs in Europe. Nevertheless, differences in physicochemical and pharmacokinetic properties statins may translate into relevant differences in long term safety. In clinical trials, all lipid-lowering agents have been associated with mild asymptomatic elevation of aminotransferase enzymes. Studies that evaluate the risk of hepatotoxicity from statins in hyperlipidemic subjects with elevated baseline serum transaminases are lacking and furthermore, statins are considered contraindicated in patients with chronic liver disease. However, clinical and biochemical monitoring is routinely recommended or required, often to make up for the lack of information on the true risk of clinically significant liver toxicity of these agents in individuals both with and without underlying liver disease although there is no evidence that monitoring reduces the rate of hepatotoxicity. This article will review what is and what is not known about hepatic disease and hepatotoxicity risk due to the statins and offers recommendations for the safe and rational use of this group of drugs in the setting of specials situations.
\end{abstract}

Keywords: Hepatotoxicity, statins, drug interactions, chronic liver disease, fatty liver.

\section{INTRODUCTION}

Cardiovascular disease (CVD) is the principal cause of death in developed countries. It is estimated that almost 17 million people world-wide die every year due to this disease [1]. Tobacco consumption, hypertension, hyperlipidemia and diabetes, are the most important risk factors for CVD [2-4] and, in combination, exacerbate the associated morbimortality [5].

Statins are potent lipid-lowering drugs which reduce the concentrations of low density lipoprotein (LDL) cholesterol by inhibiting hydroxy-methyl glutaryl coenzyme A (HMG$\mathrm{CoA}$ ) reductase (the rate limiting enzyme in the intra-cellular biosynthesis of cholesterol) mainly in liver cells. Few drugs are prescribed as often as statins and this is a result of patient tolerance to these drugs and, as well, because of their efficacy [6-8]. In Spain in 2005 alone, 27.582.700 packets were prescribed (at a cost of around $€ 727$ million) and which represented a 10\% increase over that sold in the year 2004 . Atorvastatin is the most highly prescribed statin in Spain with a $15.46 \%$ increase in sales relative to 2003 [9].

Statins are not free from side effects despite being considered as safe drugs [10]. Although infrequent, hepatotoxicity

*Address correspondence to this author at the Departamento de Farmacología, Facultad de Medicina, Boulevard Louis Pasteur, 32, Campus de Teatinos s/n, 29071 Málaga, Spain; Tel: (+34) 952 131572; Fax: (+34) 952 131568; E-mail: lucena@uma.es and myopathy are two of the most common complications associated with this class of drugs [11, 12], especially when used at maximum doses, or when combined with other lipid lowering drugs such as fibrates, or combined with drugs that use the same enzymatic pathway as cytochrome P450 (CYP450) in its metabolic pathway, or in the elderly, or in subjects having considerable hepatic and/or renal dysfunction [13-16].

Since 2004, it has been possible to obtain simvastatin 10 mg over-the-counter in the United Kingdom and a potential increase in self-medication is forecast for the population which, in view of the aforementioned clinical profile which includes asymptomatic chronic hepatopathy and hyperlipidemia, the use of this class of drug requires an alteration in the safety code assigned to it.

The aim of this article is to review the available literature regarding the hepatotoxic potential of statins and their use despite common hepatic processes such as asymptomatic hypertransaminasemia and hepatitis $\mathrm{C}$. We describe the current recommendations of their use designed to minimize the hepatotoxicity risk of these group of drugs.

\section{HEPATOTOXICITY AND DRUGS}

\section{Definition}

Hepatotoxicity is defined as the damage to the liver caused by drugs or chemicals. There are many substances capable of damaging the liver with several very different mechanisms and, as such, damage cannot be considered an 
isolated pathology but more a compounding of factors involved in its development [17].

In 1989, consensus liver injury definition was an increase greater than twice the upper limit of the normal (ULN) range of the values of serum alanine aminotransferase (ALT) or of conjugated bilirubin, or even of a combined increase in the levels of aspartate aminotransferase (AST), alkaline phosphatase (AlkPhos), and total bilirubin (TB) provided that at least one of these parameters was $>2 \times$ ULN [18]. Since 2001, an ALT level of $>3 \times$ ULN and a total bilirubin level of $>2 \times$ ULN has been used in combination to define clinically-significant abnormal liver function, with confirmation required by additional clinical and laboratory data [19]. Hence, an isolated elevated level of transaminases does not necessarily indicate hepatotoxicity, and an increase of $3 \mathrm{x}$ ULN may not be a sufficient biochemical criterion of hepatic lesion because of the considerable capacity of the liver to withstand damage [20, 21].

\section{Clinical Presentation}

The clinical presentation of hepatic toxicity is quite varied and encompasses asymptomatic or reversible alterations up to fulminant hepatic failure $[22,23]$. Further, a toxic substance may sometimes present with different patterns of toxicity and, as such, it is not always possible to link a specific clinical pattern with a particular drug [24].

To incriminate a drug in the liver symptoms observed requires a high degree of suspicion on the part of the physician and includes a temporal relationship, awareness of the drug's hepatotoxic potential, differential diagnosis and the exclusion of alternative causes of liver damage, and the ability to assess subtle data that favor a toxic etiology. Further, there are no specific analytical or clinical criteria for hepatotoxicity; exclusion of other possible etiologies being the only means of diagnosis currently available [25, 26].

Hepatotoxicity based on laboratory criteria may be classified as:

- Acute hepato-cellular type: initial elevated ALT followed by an increase in the levels of bilirubin and AlkPhos; the latter, however, being quite modest. The ALT/AlkPhos ratio (R) expressed as a function of the ULN values would be $R \geq 5$ [18] and describes patients with a presentation similar to that of acute viral hepatitis, together with general discomfort, asthenia, anorexia and nausea. Prognosis would vary substantially and be related to the extent of liver damage [27].

- Acute cholestatic injury: the initial rises are in the AlkPhos values, which are more prominent and precocious than ALT or AST and with an $R$ value $\leq 2$ [18]. The condition can be classified into two types:

Pure, soft or canalicular: clinically features include jaundice and pruritus. The condition is characterized by an increase in conjugated bilirubin, AlkPhos and $\gamma$-glutamyl transpeptidase $(\gamma$-GT) with little or no impairment in serum transaminase values. Liver histology shows hepatocyte cholestasis and dilated biliary canaliculi with bile-plugs [28]. It has a good prognosis following laboratory and clinical normalization.
Hepatocanalicular: usually presents with abdominal pain, fever and shivering; hypersensitivity episodes being frequent [18]. The condition is characterized by canalicular damage with hepatic inflammation and an increase in serum transaminases. Prognosis is better than that of hepato-cellular lesions.

- Mixed hepatic injury: is used to describe the clinical and biological picture which is intermediate between the hepato-cellular and cholestatic types, with features of either type predominating. Laboratory measurements show an elevated ALT $>2 \times$ ULN with an increment of AlkPhos and R $>2$ but $\mathrm{R}<5$ [18]. Hypersensitivity features are often present, as well as a granulomatous reaction in the liver biopsy specimen [29].

- Chronic hepato-cellular injury: information is scarce on this condition and what information is available is generally derived from isolated cases [30]. Recently, in a chronic cases study, the most frequent lesion observed was mixed/colestatic damage and the drugs most often associated with the condition were hypolipemic agents and antihypertensive medications $[31,32]$. The condition is defined by persistent elevation of the aminotransferases for $>3$ months in cases of hepato-cellular damage and $>6$ months in cholestasic/mixed case. The toxicity is, presumably, a consequence of continuing the particular treatment, or may be related to other specific drugs such as diclofenac or nitrofurantoin [33].

\section{Treatment}

In the majority of cases there is no effective treatment for the restitution of hepatic function following damage induced by a drug or its active metabolites. In the presence of either clinical or laboratory suspicion of hepatotoxicity, the first course of action is to discontinue the probable culprit substance or substances. It is important to follow-up these patients with regular monitoring of liver tests and with specialist collaboration. Clinical improvement occurs, in the majority of cases, when the culprit drug is discontinued, although in some cases the hepatotoxic reaction may well worsen weeks after the drug withdrawal, as is the case with amoxicillin-clavulanic acid [34].

\section{HEPATIC TOXICITY IN TRIALS WITH STATINS}

Abnormalities in liver function tests have been investigated in a wide range of randomized clinical trials, when comparing different doses of statins with either placebo or another comparator (Table 1). In a meta-analysis of randomized clinical trials (RCTs) of fluvastatin, pravastatin, lovastatin and simvastatin at low or moderate doses $(<40 \mathrm{mg}$ oral), the prevalence of elevated transaminases $>3 \times$ ULN was similar in the active treatment groups compared to the placebo groups, but only slightly higher in two studies of fluvastatin versus placebo $(1.13 \%$ vs $0.39 \%, \mathrm{p}=0.04)$ [35]. However, atorvastatin despite being the most-prescribed statin had not been included in this meta-analysis. When analyzing large-scale RCTs where high-dose atorvastatin was used ( $80 \mathrm{mg}$ oral), the prevalence of asymptomatic elevations of transaminases $>3 \times$ ULN was slightly higher $(\approx 3 \%)$ compared to placebo, or with another statin at moderate dose (Table 2) [36-39]. In comparing the effects of $10 \mathrm{mg}$ versus $80 \mathrm{mg}$ daily atorvastatin, it appears that the frequency 
of an abnormal transaminase value is $0.6 \%$ for the $10 \mathrm{mg}$ dose, increasing up to $3.3 \%$ for the $80 \mathrm{mg}$ dose [40, 41]. This safety profile is maintained even in individuals $>65$ years of age [42]. Hence, in summary, the risk of hypertransaminasemia or acute hepatitis associated with these drugs as seen in the clinical trials, is no higher than the risk in the placebo group when using the low or moderate doses.

Table 1. Percentages of Transaminase Increases in Patients Treated with Placebo and Different Dose of Statins (Denus 2004) [35]

\begin{tabular}{|c|c|c|c|c|c|}
\hline \multirow{2}{*}{ Statin } & \multirow{2}{*}{ Placebo } & \multicolumn{4}{|c|}{ STATIN Dose } \\
\cline { 3 - 6 } & & $\mathbf{1 0} \mathbf{~ m g}$ & $\mathbf{2 0} \mathbf{~} \mathbf{2 g}$ & $\mathbf{4 0} \mathbf{~} \mathbf{8}$ & $\mathbf{8 0} \mathbf{~ m g}$ \\
\hline \hline Lovastatin & $0.1 \%$ & - & $0.1 \%$ & $0.9 \%$ & $2.3 \%$ \\
\hline Simvastatin & - & - & $0.7 \%$ & $0.9 \%$ & $2.3 \%$ \\
\hline Pravastatin & $1.3 \%$ & - & - & $1.4 \%$ & - \\
\hline Fluvastatin & $0.3 \%$ & - & $0.2 \%$ & $1.5 \%$ & $2.7 \%$ \\
\hline Atorvastatin & - & $0.2 \%$ & $0.2 \%$ & $0.6 \%$ & $2.3 \%$ \\
\hline
\end{tabular}

An Alanine transaminase increases superior to three times the upper limit of normality.

Nonetheless, in large-scale RCTs with statins, an exclusion criterion is the presence of hypertransaminasemia or active liver disease and, therefore, the hepatotoxic risks that these drugs can provoke in standard clinical practice are not assessed. A Medline search (dated October $5^{\text {th }}$ 2007) using acute hepatitis and statins as MeSH terms revealed only 37 original entries reporting cases of acute hepatitis caused by these drugs. Further, in a national registry for drug-related hepatic reactions in which 461 episodes where recorded, only $11(2.3 \%)$ were attributable to statins [31]. These data confirm the low incidence of severe adverse hepatic reactions provoked by the use of $\mathrm{HMG}-\mathrm{CoA}$ reductase inhibitors.

\section{STATINS AND COMMON CLINICAL SITUATIONS}

\section{Asymptomatic Hypertransaminasemia}

In standard clinical practice it is not infrequent to prescribe statins for patients with asymptomatic hypertransaminasemia. Once viral infection has been excluded, the majority of these subjects are described as having "fatty liver" [43], an entity that has become the most frequent hepatopathy in the Western World and, probably, in Spain as well $[44,45]$. Indeed, the condition has reached a prevalence of $13.8 \%$ according to a work-place study conducted with 1713 Spanish males aged between 18 and 60 years; prevalence was reduced to $8.8 \%$ when obese and diabetic subjects ere excluded form the analyses [46]. Further, non-alcoholic fatty liver or non-alcoholic fatty liver disease (NAFLD) was clearly associated with abdominal obesity, dyslipemia and diabetes mellitus, all of what are risk factors for atherosclerosis [47]. Several longitudinal studies have revealed that cardiovascular disease is the leading cause of death in subjects suffering from NAFLD, followed by chronic liver disease [48]. Also, NAFLD is associated with the Metabolic Syndrome (Table 3) [49], an entity which, based on the guidelines of the National Cholesterol Education ProgramAdult treatment Panel III (NCEP-ATP III), increases the predisposition to cardiovascular disease [50, 51]. Indeed, approximately $80 \%$ of patients with non-alcoholic steatohepatitis, as confirmed by biopsy, fulfill the criteria for the diagnosis of Metabolic Syndrome [52].

Statins being the drugs-of-choice in the treatment of hypercholesterolemia and, as well, in the prevention of cardiovascular disease, it would be of interest to evaluate whether the presence of hypertransaminasemia could lead to a poorer prognosis if the patients receive treatment with statins. In this respect the publications by Chalasani et al. [53] and by Vuppalanchi et al. [54] are of considerable interest. Chalasani et al. retrospectively evaluated the hepato-toxicity risk in subjects treated for at least 6 months with atorvastatin, simvastatin or fluvastatin. Drawn from the pharmacological registry of an insurance company, the subjects were segregated into two groups: one group with elevated transaminases before treatment (cohort I; $\mathrm{n}=342$ ) and another group with normal levels of transaminases (cohort II; $n=$ 1435). Both groups were compared with a third group with hypertransaminasemia but not receiving lipid-lowering drugs (cohort III, n=2245). Alcoholic or viral etiology was excluded in all of the three groups. The authors' criteria for "low-moderate elevation" was an increase of ALT and/or AST $<10 \times$ baseline values. By contrast, an increase of ALT and/or AST $>10 \times$ baseline value or total bilirubin values $>3$ $\mathrm{mg} / \mathrm{dL}$, were considered as "severe reaction". Incidence of low-moderate elevations was $4.7 \%, 1.9 \%$ and $6.4 \%$, respectively, in the three cohorts $(p<0.05)$ while incidence of severe reactions was $0.6 \%, 0.2 \%$ and $0.4 \%(\mathrm{p}>0.2)$. The authors concluded that patients who already have moderate hypertransaminasemia before starting the treatment are not at higher risk of statin-induced hepatotoxicity.

Another more recent study by Vuppalanchi et al. [54] analyzed the effects of 1 year treatment with lovastatin. The three cohorts evaluated followed the same criteria used by Chalasani et al. The number of participants in each group was 135 in cohort I, 620 in cohort II and 2644 patients in cohort III. The study had an additional hepatic lesion criterion introduced. Termed the Hy's rule, the criterion is based on the presence of ALT and/or AST >3 x ULN, plus biliru-

Table 2. Frequency of Abnormal Liver Function Test (ALT >3 x ULN) in Studies with Atorvastatin Relative to its Comparator

\begin{tabular}{|c|c|c|c|c|c|c|}
\hline Study (Year) & Number of Patients & Atorvastatin mg Dose & \%ALT & Comparator & \%ALT & Statistical Significance \\
\hline \hline CARDS (2004) & 2838 & 10 & 0.4 & Placebo & 0.3 & NS \\
\hline MIRACL (2001) & 3086 & 80 & 2.5 & Placebo & 0.6 & $<0.05$ \\
\hline REVERSAL (2005) & 654 & 80 & 2.3 & Pravastatin 40 & 1.6 & 1.1 \\
\hline PROVE-IT (2004) & 3745 & 80 & 3.3 & Pravastatin 40 & NS & $<0.05$ \\
\hline SPARCL (2006) & 4731 & 80 & 2.2 & Placebo & 0.4 & $<0.05$ \\
\hline
\end{tabular}


Table 3. Main Analytical Characteristics of the Metabolic Syndrome

\begin{tabular}{|c|c|c|c|}
\hline Lipoproteins & Glycemia & Blood Pressure & Waist Circumference \\
\hline \hline Triglycerides $\geq 150 \mathrm{mg} / \mathrm{dL}$ & & & $>102 \mathrm{~cm}(\mathrm{Males})$ \\
High density lipoprotein: & & $\geq 130 / 85 \mathrm{mmHg}$ & $>88 \mathrm{~cm}(\mathrm{Females})$ \\
$<40 \mathrm{mg} / \mathrm{dL}$ (Males) & glucose $\geq 110 \mathrm{mg} / \mathrm{dL}$ & \\
\hline $50 \mathrm{mg} / \mathrm{dL}$ (Females) & & & \\
\hline
\end{tabular}

bin $>2 \times$ ULN in the course of the observation period, and which is considered as clinically-evident hepatitis, with a $10 \%$ mortality [55]. Results were super-imposable on those obtained in the study by Chalasani et al. Of note is that none of the patients in cohorts I and II matched the Hy's criteria, while its prevalence was $3 \%$ in cohort III. It is clear that a substantial proportion of patients $(4.6 \%$ from cohort I and $4.5 \%$ from cohort II of the initial study) showed ALT and/or AST $>3 \times$ ULN. Since this event is considered a benign hypertransaminasemia it has become usual practice to reduce, or discontinue, treatment with statins and which, in the short term, results in a reduction in therapeutic efficiency.

Despite that the majority of patients from the two studies mentioned above suffering from NAFLD and, as well, that a non-controlled study suggested an analytic benefit (reductions in aminotrasferase, AlkPhos, and $\gamma$-GT) in NAFLD patients treated with atorvastatin $10 \mathrm{mg}$ [56], there have not been any randomized clinical trials that support the conclusion that the use of statins in this clinical context would provide benefit with respect to the resolution of histological lesions and protection against the progression of the disease towards fibrosis and cirrhosis.

\section{Patients with Chronic Hepatitis $\mathrm{C}$}

Hepatitis $\mathrm{C}$ virus (HCV) infection is the most frequent cause of long-term hepatic lesion. Nearly 170 million people world-wide are infected with the virus, most being asymptomatic carriers with minimal change in the levels of the hepatic enzymes $[57,58]$. Because of its high incidence, it is not unusual to find patients with chronic HCV and hyperlipidemia being treated with lipid lowering drugs, mainly statins.

HCV patients have been excluded from clinical trials with statins because of the perceived increased risk of hepatic toxicity. However, two recent studies have shown an absence of significant liver enzyme elevation in infected versus control groups. In an early review [59] of 219 patients with chronic $\mathrm{HCV}, 17$ were receiving statin treatment for at least 3 months. Only 5 patients had any liver enzyme elevation while on a statin, and the highest increase was $1.5 \mathrm{x}$ ULN. A subsequent study [60] followed 3 cohorts of patients for 12 months and compared the values of the transaminases in follow-up with those recorded at baseline. Cohort 1 was composed of 166 patients with hyperlipidemia and $\mathrm{HCV}$ positive and had statins administered; Cohort II was composed of 332 patients with $\mathrm{HCV}$-antibody positive and were not receiving statins treatment; Cohort III was composed of 332 patients with negative HCV antibodies and hyperlipidemia and receiving statins. When cohort I and II were compared, a higher frequency of moderate elevations of aminotrasferases $(23 \%$ vs $13 \%$; $\mathrm{p}<0.05)$ was observed as well as, paradoxically, a lower frequency of acute elevations
$(1.2 \%$ vs $6.6 \% ; \mathrm{p}<0.05)$ in cohort I compared to cohort II. When comparing cohorts I and III, the moderate increase appeared very similar for both groups $(22.9 \%$ vs $16.3 \%$; $\mathrm{p}=0.094)$ as did the severe increase $(1.2 \%$ vs $1 \% ; \mathrm{p}=0.874)$ and when the hepatotoxic treatment was withdrawn $(21.6 \%$ vs $9.2 \%$; $\mathrm{p}=0.147)$. The conclusion was that lipid lowering therapy with statins in patients with chronic HCV is not associated with a significant increase in hepatotoxicity risk [59]. However, additional studies would be necessary to corroborate these findings, particularly in cases of severe infection.

The standard treatment for hepatitis $\mathrm{C}$ patients is based on the combination of pegylated interferon and ribavirin, although the effectiveness is limited to approximately 55\% of patients [61]. Lovastatin appears to inhibit HCV RNA replication by inhibiting the geranylgeranylation of cellular proteins rather than the inhibition of cholesterol synthesis [62]. A recent publication of Ikeda et al. reported that lovastatin, fluvastatin, atorvastatin and simvastatin, but not pravastatin, inhibit HCV RNA replication in in vitro cell culture [57]. Further, all statins, except pravastatin, markedly enhanced the anti-HCV effect of IFN- $\alpha$ [57]. Differences observed between pravastatin and other statins could be related to pravastatin's absence of metabolic processes via cytochrome P450 (Table 4) [63, 64].

\section{Patients with Primary Biliary Cirrhosis}

Primary biliary cirrhosis is a chronic progressive cholestatic disorder characterized by a marked increase of cholesterol, mainly due to $\mathrm{Lp} \mathrm{X}$ particles, that may elevate the risk of coronary heart disease [65]. Two small trials evaluated the role of simvastatin [66] and atorvastatin [67], indicating that both drugs were able to reduce total cholesterol as expected; however, no clear improvement in cholestatic parameters were observe It is worth pointing out that these trials included only 21 patients followed-up for just 6 and 8 weeks. In summary, additional larger scale, prospective studies are required to determine the efficacy of statins in this population.

\section{CONCLUSIONS AND RECOMMENDATIONS}

Statins are the first-line therapy for patients with dyslipidemia involving elevated LDL, and for the prevention of cardiovascular disease. Despite being among the most widely prescribed drugs on the market, there is a dearth of reported cases of hepatotoxicity. The frequency of elevated aminotrasferase is $<1 \%$ for low-to-moderated doses $(\leq 40$ $\mathrm{mg}$ ) and reaches $3 \%$ for high doses $(80 \mathrm{mg})$. Therefore, the risk of suffering from liver failure as a consequence of this class of drugs, is low i.e. approximately about 1 per million, according to notification to regulatory authorities [12]. 
Table 4. Pharmacokinetic Parameters of Statins

\begin{tabular}{|c|c|c|c|c|c|}
\hline & Lovastatin & Simvastatin & Pravastatin & Fluvastatin & Atorvastatin \\
\hline Lipophilic & Yes & Yes & No & Yes & Yes \\
\hline Protein binding & $>95 \%$ & $95-98 \%$ & $50 \%$ & $>98 \%$ & $98 \%$ \\
\hline CYP450 & CYP3A4 & CYP3A4 & No & CYP2C9 & CYP3A4 \\
\hline Half life & $3 \mathrm{~h}$ & $2 \mathrm{~h}$ & $1.8 \mathrm{~h}$ & $1.2 \mathrm{~h}$ & $1.4 \mathrm{~h}$ \\
\hline Active metabolites & Yes & Yes & No & No & Yes \\
\hline $\mathrm{Bd}$ & $5 \%$ & $5 \%$ & $18 \%$ & $19-30 \%$ & $12 \%$ \\
\hline Urinary Excretion & $10 \%$ & $13 \%$ & $20 \%$ & $6 \%$ & $2 \%$ \\
\hline Tmax & 2-4 hour & 1.3-2.4 hour & 0.9-3.6 hour & $0.5-1$ hour & 2-3 hour \\
\hline
\end{tabular}

Bd: Bio-availability; Tmax: time to peak concentration.

The evidence that patients with baseline hypertransaminasemia are not at a higher risk of hepatotoxicity when receiving statins than the general population prompts the consideration these drugs are beneficial in the treatment vascular risk associated to NAFLD. None of the current studies would formally contraindicate the prescription of statins for patients with hepatitis $\mathrm{C}$, although we should keep in mind the low number of participant in such studies. Further, the use of this class of drugs in combination with interferon to improve the conventional treatment efficiency is currently being investigated.

As already stated, there is no evidence that statin therapy should be altered or discontinued solely on the basis of elevated aminotransferase levels in an asymptomatic patient. Should more objective evidence of hepatic dysfunction be identified, such as hepato-megaly, clinical evidence of jaundice, elevated direct bilirubin, or increased prothrombin time, discontinuation of statin therapy ought to be considered [68]. In the absence of biliary obstruction, the plasma concentration of bilirubin is a more reliable prognostic factor of liver injury, in the drug toxicity setting. If the direct fraction of bilirubin is found to be increased together with elevated aminotransferases, it is reasonable to assume that there is ongoing liver injury and further appropriate laboratory and clinical evaluation should be undertaken to ascertain the etiology.

Once a patient recovers from a hepatotoxic episode induced by statins, it is worthy to re-evaluate the need for cholesterol-lowering drugs. If this is the case, in addition to lowcholesterol and low-saturated fat diet, the alternatives would be resins or ezetimibe. In the subset of patients with a very high vascular risk (i.e. diabetics with coronary heart disease already present), then it would be necessary to switch from one statin group to another; for instance from a prolactone drug (simvastatin or lovastatin) to a synthetic one (atorvastatin or pravastatin) or vice-versa. Careful evaluation of the risk/benefit ratio and close monitoring should be followed.

\section{ACKNOWLEDGEMENT}

CIBERehd is funded by Instituto de Salud Carlos III.

\section{REFERENCES}

[1] Parra JL, Reddy KR. Hepatotoxicity of hypolipidemic drugs. Clin Liver Dis 2003; 7 (2): 415-33.
[2] Wang TJ, Gona P, Larson MG, et al. Multiple biomarkers for the prediction of first major cardiovascular events and death. N Engl J Med 2006; 355(25): 2631-9.

[3] Sander GE, Giles TD. Hypertension and lipids: lipid factors in the hypertension syndrome. Curr Hypertens Rep 2002; 44(66): 458-63.

[4] Toth PP. High-density lipoprotein as a therapeutic target: clinical evidence and treatment strategies. Am J Cardiol 2005; 96(9A): $50 \mathrm{~K}-8 \mathrm{~K}$.

[5] Brown BG, Zhao X-Q, Chait A, et al. Simvastatin and niacin, antioxidant vitamins, or the combination for the prevention of coronary disease. N Engl J Med 2001; 345(22): 1583-92.

[6] Shepherd J. The West of Scotland Coronary Prevention Study: a trial of cholesterol reduction in Scottish men. Am J Cardiol 1995; 76(9): 113C-7C.

[7] Maron D, Fazio S, Linton M. Current perspectives on statins. Circulation 2000; 101(2): 207-13.

[8] Maddrey W. Drug Induced Hepatotoxicity. J Clin Gastroenterol 2005; 39: S83-S9.

[9] Subgrupos ATC. y Principios activos de mayor consumo en el SNS en 2005. Inf Ter SNS 2006; 2: 42-9.

[10] Hsu I, Spinler SA, Johnson NE. Comparative evaluation of the safety and efficacy of HMG-CoA reductase inhibitor monotherapy in the treatment of primary hypercholesterolemia. Ann Pharmacother 1995; 29(7-8): 743-59.

[11] Pasternak RC, Smith SCJ, Bairey-Merz CN, et al. ACC/AHA /NHLBI Clinical Advisory on the Use and Safety of Statins. Circulation 2002; 106(8): 1024-8.

[12] Law M, Rudnicka AR. Statin safety: a systematic review. Am J Cardiol 2006; 97(8A): 52C-60C

[13] Martinez TL, Nascimento HM. [Special recommendations for lipid-lowering treatment: efficacy and safety]. Arq Bras Cardiol 2005; 85 (Suppl 5): 6-8.

[14] Kajinami K, Akao H, Polisecki E, Scahefer EJ. Pharmacogenomics of statin responsiviness. Am J Cardiol 2005; 96 (9A): 65K-70K.

[15] Bottorff MB. Statin safety and drug interactions: clinical implications. Am J Cardiol 2006; 97(8 S1): S27-S28.

[16] Talbert R. Safety issues with statin therapy. J Am Pharm Assoc 2006; 46(4): 479-88.

[17] Kaplowitz N. Idiosyncratic drug hepatotoxicity. Nat Rev Drug Discov 2005; 4(6): 489-99.

[18] Benichou C. Criteria for drug-induced liver disorders. Report of an International Consensus Meeting. J Hepatol 1990; 11: 272-276.

[19] CDER-PH RMA. AASLD Conference 2000: clinical white paper on drug-induced hepatotoxicity. (www.fda.gov/cder/livertox/clinical.pdf).

[20] Hardisty JF, Brix AE. Comparative hepatic toxicity: prechronic/chronic liver toxicity in rodents. Toxicol Pathol 2005; 33(1): 35-40.

[21] Navarro VJ, Senior JR. Drug-related Hepatotoxicity. N Engl J Med 2006; 354: 731-9.

[22] Kaplowitz N, Aw TY, Simon FR, Stolz A. Drug-induced hepatotoxicity. Ann Intern Med 1986; 104(6): 826-39.

[23] Bertolami MC. [Mechanisms of hepatotoxicity]. Arq Bras Cardiol 2005; 85 (Suppl 5): 25-7.

[24] Knowles SR UJ, Shear NH. Idiosyncratic drug reactions: the reactive metabolite syndromes. Lancet 2000; 356: 1587-91. 
[25] Lee WM. Drug-induced hepatotoxicity. N Engl J Med 1995; 333 : 1118-27.

[26] Kaplowitz N. Rules and laws of drug hepatotoxicity. Pharmacoepidemiol Drug Saf 2006; 15: 231-3.

[27] Ostapowicz G, Fontana RJ, Schiodt FV, et al. Results of a prospective study of acute liver failure at 17 tertiary care centers in the United States. Ann Intern Med 2002; 137: 947-54.

[28] Kaplowitz N. Biochemical and cellular mechanisms of toxic liver injury. Sem Liver Dis 2002; 22: 137-44.

[29] Larrey D, Erlinger S. Drug- induced cholestasis. Clin Gastroenterol 1988; 2: 423-52.

[30] Andrade RJ, Lucena MI, Kaplowitz N, et al. Outcome of acute idiosyncratic drug-induced liver injury: long-term follow-up in a hepatotoxicity registry. Hepatology 2006; 44: 1581-8.

[31] Andrade RJ, Lucena MI, Fernandez MC, et al. Drug-induced liver injury: an analysis of 461 incidences submitted to the Spanish registry over a 10-year period. Gastroenterology 2005; 129(2): 512-21.

[32] Chalasani N. Statins and hepatotoxicity: focus on patients with fatty liver. Hepatology 2005; 41(4): 690-5.

[33] Farrell GC. Drug induced liver disease. London: ChurchillLivingstone: 1994.

[34] Zimmerman HJ. Hepatotoxicity: the adverse effects of drugs and other chemicals on the liver. Philadelphia. Lippincot Williams \& Wilkins, 1999.

[35] Denus S, Spinler SA, Miller K, Peterson AM. Statins and Liver toxicity: A meta-analysis. Pharmacotherapy 2004; 24: 584-91.

[36] Schwartz GG, Olsson A, Ezekowitz M, et al. Myocardial Ischemia Reduction with Aggressive Cholesterol Lowering (MIRACL) Study Investigators. Effects of Atorvastatin on Early Recurrent Ischemic Events in Acute Coronary Syndromes The MIRACL Study: A Randomized Controlled Trial. JAMA 2001; 285(13): 1711-8.

[37] Nissen SE, Tuzcu EM, Schoenhagen P, et al. Effect of intensive compared with moderate lipid-lowering therapy on progression of coronary atherosclerosis: a randomized controlled trial. JAMA 2004; 291 (9): 1071-80.

[38] Colhoun HM, Betteridge DJ, Durrington PN, et al. Primary prevention of cardiovascular disease with atorvastatin in type 2 diabetes in the Collaborative Atorvastatin Diabetes Study (CARDS): multicentre randomised placebo-controlled trial. Lancet 2004; 364(9435): 685-96.

[39] Amarenco P, Bogousslavsky J, Callahan A3, et al. Stroke Prevention by Aggressive Reduction in Cholesterol Levels (SPARCL) Investigators. High-dose atorvastatin after stroke or transient ischemic attack. N Engl J Med 2006; 355(6): 549-59.

[40] Newman AB, Shemanski L, Manolio TA, et al. Ankle-arm index as a predictor of cardiovascular disease and mortality in the cardiovascular health study. Am J Cardiol 1999; 19(3): 538-45.

[41] Newman C, Tsai J, Szarek M, Luo D, Gibson E. Comparative safety of atorvastatin $80 \mathrm{mg}$ versus $10 \mathrm{mg}$ derived from analysis of 49 completed trials in 14,236 patients. Am J Cardiol 2006; 97(1): 61-7.

[42] Hey-Hadavi JH, Kuntze E, Luo D, Silverman P, Pittman D, Lepetri B. Tolerability of atorvastatin in a population aged $>$ or $=65$ years: a retrospective pooled analysis of results from fifty randomized clinical trials. Am J Geriatr Pharmacother 2006; 4(2): 112-22.

[43] Cuadrado A, Crespo J. Hypertransaminasemia in patients with negative viral markers. Rev Esp Enferm Dig 2004; 96(7): 484-92.

[44] Angulo P. Treatment of nonalcoholic fatty liver disease. Ann Hepatol 2002; 1(1): 12-9.

[45] Angulo P. Nonalcoholic fatty liver disease. N Engl J Med 2002; 346(16): 1221-31.

[46] Pares A, Tresserras R, Nunez I, et al. [Prevalence and factors associated to the presence of fatty liver in apparently healthy adult men]. Med Clin 2000; 114(15): 561-5.

[47] Brea A, Mosquera D, Martin E, Arizti A, Cordero JL, Ros E. Nonalcoholic fatty liver disease is associated with carotid Atherosclero- sis: A Case-control Study. Arterioscler Thromb Vasc Biol 2005; 25(5): 1045-50.

[48] Adams LA, Lymp JF, St Sauver J, et al. The natural history of nonalcoholic fatty liver disease: a population-based cohort study. Gastroenterology 2005; 129(1): 113-21.

[49] Third Report of the National Cholesterol Education Program (NCEP) Expert Panel on Detection, Evaluation, and Treatment of High Blood Cholesterol in Adults (Adult Treatment Panel III) Final Report. Circulation 2002; 106(25): 3143.

[50] Haffner SM, Lehto S, Ronnemaa T, Pyorala K, Laakso M. Mortality from coronary heart disease in subjects with type 2 diabetes and in nondiabetic subjects with and without prior myocardial infarction. N Engl J Med 1998; 339(4): 229-34.

[51] Lorenzo C, Williams K, Hunt KJ, Haffner SM. The National Cholesterol Education Program-Adult Treatment Panel III, International Diabetes Federation, and World Health Organization Definitions of the Metabolic Syndrome as Predictors of Incident Cardiovascular Disease and Diabetes. Diabetes Care 2007; 30(1): 8-13.

[52] Marchesini G, Bugianesi E, Forlani G, et al. Nonalcoholic fatty liver, steatohepatitis, and the metabolic syndrome. Hepatology 2003; 37(4): 917-23.

[53] Chalasani N, Aljadhey H, Kesterson J, Murray MD, Hall SD. Patients with elevated liver enzymes are not at higher risk for statin hepatotoxicity. Gastroenterology 2004; 126(5): 1287-92.

[54] Vuppalanchi R, Teal E, Chalasani N. Patients with elevated baseline liver enzymes do not have higher frequency of hepatotoxicity from lovastatin than those with normal baseline liver enzymes. Am J Med Sci 2005; 329(2): 62-5.

[55] Reuben A. Hy's law. Hepatology 2004; 39(2): 574-8.

[56] Kiyici M, Gulten M, Gurel S, et al. Ursodeoxycholic acid and atorvastatin in the treatment of nonalcoholic steatohepatitis. Can J Gastroenterol 2003; 17(12): 713-8.

[57] Ikeda M, Abe K, Yamada M, Dansako H, Naka K, Kato N. Different anti-HCV profiles of statins and their potential for combination therapy with interferon. Hepatology 2006; 44(1): 117-25.

[58] Tolman KG. Defining patient risks from expanded preventive therapies. Am J Cardiol 2000; 85(12A): 15E-9E.

[59] Gibson K, Rindone JP. Experience with statin use in patients with chronic hepatitis C infection. Am J Cardiol 2005; 96(9): 1278-9.

[60] Khorashadi S, Hasson NK, Cheung RC. Incidence of statin hepatotoxicity in patients with hepatitis C. Clin Gastroenterol Hepatol 2006; 4(7): 902-7.

[61] Degott C, Larrey D, Duran-Schneider AM, et al. Drug-induced prolonged cholestasis in adults: a histological semiquantitative study demonstrating progressive ductopenia. Hepatology 1992; 15: 244-51.

[62] Kapadia SB, Chisari FV. Hepatitis C virus RNA replication is regulated by host geranylgeranylation and fatty acids. Proc Natl Acad Sci USA 2005; 102(7): 2561-6.

[63] Bolego C, Baetta R, Bellosta S, Corsini A, Paoletti R. Safety considerations for statins. Curr Opin Lipidol 2002; 13(6): 637-44.

[64] Kocarek TA, Dahn MS, Cai H, Strom SC, Mercer-Haines NA. Regulation of CYP2B6 and CYP3A expression by hydroxymethylglutaryl coenzyme A inhibitors in primary cultured human hepatocytes. Drug Metab Dispos 2002; 30(12): 1400-5.

[65] Sorokin A, Brown JL, Thompson PD. Primary biliary cirrhosis, hyperlipidemia, and atherosclerotic risk: a systematic review. Atherosclerosis 2007; 194(2): 293-9.

[66] Ritzel U, Leonhardt U, Nather M, Schafer G, Armstrong VW, Ramadori G. Simvastatin in primary biliary cirrhosis: effects on serum lipids and distinct disease markers. J Hepatol 2002; 36(4): 454-8.

[67] Stojakovic T, Putz-Bankuti C, Fauler G, et al. Atorvastatin in patients with primary biliary cirrhosis and incomplete biochemical response to ursodeoxycholic acid. Hepatology 2007; 46(3): 776-84.

[68] Cohen DE, Anania FA, Chalasani N. An assessment of statin safety by hepatologists. Am J Cardiol 2006; 97(8A): 77C-81C. 